\title{
Kinetic real-space renormalization-group approach to the shortest-path aggregation
}

\author{
Xiang Rong Wang \\ Department of Physics, Hong Kong University of Science and Technology, Kowloon, Hong Kong
}

(Received 10 September 1993)

\begin{abstract}
A kinetic real-space renormalization-group approach to the shortest-path aggregation (SPA) is presented. The fractal dimension $\left(D_{f}\right)$ and a set of hierarchical dimensions $D(q)$ are computed for the SPA cluster on the square lattice. In particular, $2 \times 2$ and $3 \times 3$ cell real-space renormalization groups have been carried out. We find $D_{f}=1.19$ and 1.21 , respectively, in good agreement with the simulations.
\end{abstract}

PACS number(s): 68.70. $+\mathrm{w}, 05.40 .+\mathrm{j}, 82.20 .-\mathrm{w}$

In the past decade, much interest has been devoted to the irreversible aggregation of complex structures from small subunits. It was recognized that many important kinetic aggregation processes result in fractal geometry, such as electron deposition and viscous fingering [1]. To study the mechanism of fractal pattern formation, several models have been considered in recent years, such as the diffusion-limited aggregation (DLA) model [2] and the ballistic aggregation model [3]. In particular, an irreversible kinetic aggregation lattice model called the shortest-path aggregation (SPA) has been introduced [4]. Unlike DLA, diffusion is no longer the mechanism in this model. Similar to the DLA model, the SPA model is highly unstable [4]. This is clear from definition of the model: A seed is placed in a lattice. Particles released from the randomly chosen distant sites will deposit on a certain part of the cluster along the shortest Manhattan path [4].

It is clear from the model that the screening effect in this model is so strong that each tip of the cluster will completely overshadow a certain region, prohibiting growth, while growth in such a region is still allowed in the DLA model (though it is small). Due to this strong screening effect, this model generates noncompact clusters with fractal dimension much smaller than that of the DLA, and at the same time it is simpler than the latter because of its more deterministic deposition path. The simulations show that the shortest-path aggregation generates the clusters with fractal dimension being $D \approx 1.21$ in two dimensions (2D) [4]. There are also numerical evidences that there is a universal behavior of the cluster formation in the sense that the fractal dimensions are the same when the released particles are weakly correlated as that when the released particles are independent of each other [4].

It will be interesting to study the shortest-path aggregation analytically. One of the natural approaches is the real-space RG method because of the fractal structure of the SPA clusters. In fact, the real-space RG approaches had been applied to diffusion-limited aggregation [6-8]. In particular, Wang, Shapir, and Rubinstein [6] have recently proposed a kinetic real-space renormalization (KRG) approach in which the kinetic growth determines the recursion relations for the probabilities of the configuration on consecutive scales. The approach has been applied to the DLA model successfully with very good results. It has been further shown by Wang and Huang [9] that this method is exact for DLA in infinite space.

Following the kinetic renormalization-group approach to DLA [6], we will apply it to calculate the fractal and multifractal dimensions for SPA in two-dimensional space. The KRG approach is based on the combined time and length scale invariance of the SPA cluster, which is used to obtain the relation between the probabilities for configurations on consecutive scales directly from the growth process itself. Indeed, given a cluster configuration at time $t$, probabilities for the configuration at time $t+1$ (with one more particle) are completely defined from the growth probabilities on the surface sites. The KRG approach uses the fact that the growth probability distribution remains invariant and is the same at $t+1$ as it was at $t$. In practice, we can only find the recursion relations between probabilities of the configurations of small cells, like the one we choose here depicted in Fig. 1.

In the lattice-bond version of the SPA, using the KRG approach of Wang et al., we thus distinguish between three types of bonds at each order $n$ of the transformation: bulk bonds representing the particles on the cluster with mass $M_{n}$ (bold lines in Fig. 1); perimeter bonds with mass $m_{n}$ (wavy lines), representing the surface of the aggregate on which the next potential growth may occur; and massless empty bonds for the rest (thin lines).

A cluster grows by changing perimeter bonds to bulk bonds one by one according to probabilities which are determined by the growth mechanism. As a particle is added to the cluster one perimeter bond turns into a bulk bond and all its unoccupied neighbors become growth bonds. The following rules guide the transformation: If the cell is connected from top to bottom by bulk bonds it will be renormalized into a bulk bond; if the cell is completely empty it will be renormalized to an empty bond; all other configurations are renormalized into a perimeter bond. Therefore all configurations in Fig. 1(a) will be renormalized to the vertical perimeter bonds, and all configurations in Fig. 1(b) will be renormalized to vertical bulk bonds.

In order to find the local growth probabilities on each bond, we notice that the particles are released from the top and adhere to perimeter bonds along the shortest path, and, if there is more than one such path for a particular particle, then each path has an equal chance to be chosen. Let us take configuration 1 of Fig. 1(a) an an example. If we denote $p_{1,1}$ and $p_{1,2}$ as the growth probabilities on the perimeter bonds 1 and 2 , respectively. Ac- 

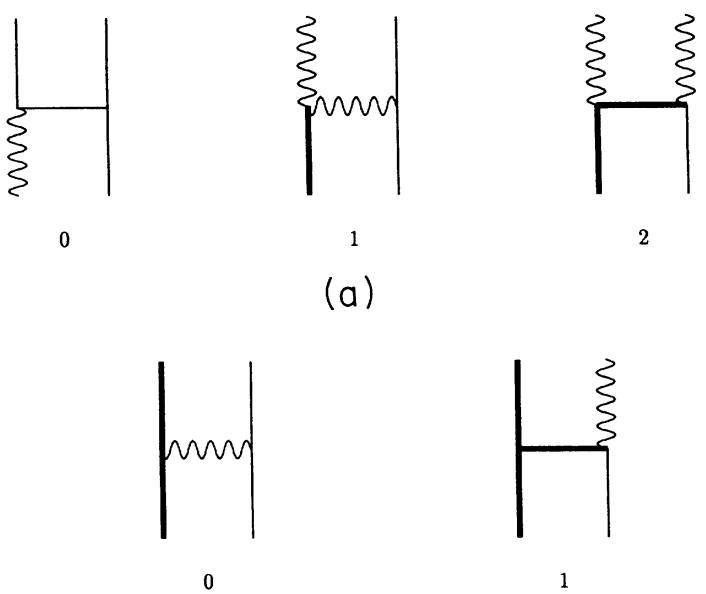

(a)

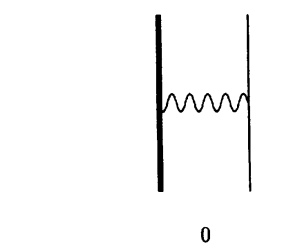

\section{(a)}

(a)

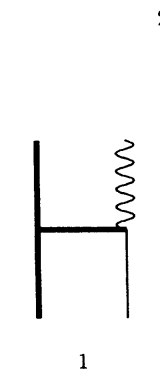

(b)

FIG. 1. (a) All possible configurations which are renormalized to the vertical perimeter bonds for the square lattice in a $2 \times 2$ cell. (b) All possible configurations which are renormalized to the vertical bulk bonds for the square lattice in a $2 \times 2$ cell.

cording to the growth mechanism, a particle has equal probabilities to be released from the top left and top right sides. Obviously, if the particle is released from the top left side, perimeter bond 1 will be converted into a bulk bond because perimeter bond 1 is the closest perimeter bond from the released particle. On the other hand, the particle will be deposited on perimeter bond 2 if it is released from the top right side. Therefore $p_{1,1}=p_{1,2}=0.5$. Similarly, we obtain the growth probabilities of all the configurations in Fig. 1(a).

To find the probability $C_{\alpha}$ for each configuration in Fig. 1(a) to appear on the surface of the aggregate, we notice that configuration 1 is generated from configuration 0 by one step of the SPA growth-by adding one bulk bond to bond 1 of configuration 0 , and replacing the two empty bonds next to the added one by the perimeter bonds, so

$$
C_{1}=p_{0,1} C_{0} \text {. }
$$

Configuration 2 is generated from configuration 1 by adding one bulk bond to the bond 2 of configuration 1, such that

$$
C_{2}=p_{1,2} C_{1} \text {. }
$$

Thus

$$
C_{1}=C_{0}, \quad C_{2}=0.5 C_{1},
$$

with the normalization condition

$$
\sum_{\alpha} C_{\alpha}=1
$$

The resulting configuration weights $C_{\alpha}$ and the growth probabilities $p_{\alpha, i}$ are presented in Table I. Notice that the probabilities $C_{\alpha}$ of various configurations were relat-

TABLE I. Values of $C_{\alpha}$ and $p_{\alpha, i}(2 \times 2$ cell $)$.

\begin{tabular}{cccc}
\multicolumn{5}{c}{ TABLE I. Values of $C_{\alpha}$ and $p_{\alpha, i}(2 \times 2$ cell $)}$. \\
\hline \hline$\alpha$ & $C_{\alpha}$ & $p_{\alpha, 1}$ & $p_{\alpha, 2}$ \\
\hline 0 & 0.4 & 1 & 0 \\
1 & 0.4 & 0.5 & 0.5 \\
2 & 0.2 & 0.5 & 0.5 \\
\hline \hline
\end{tabular}

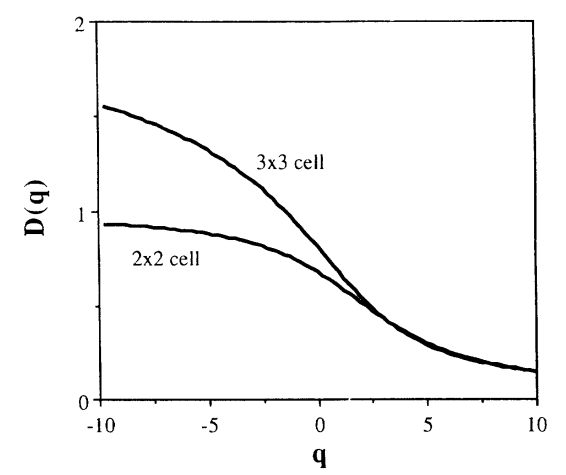

FIG. 2. The plot of $D(q)$ vs $q$ for the SPA on the square lattice for $2 \times 2$ and $3 \times 3$ cell renormalizations.

ed to each other by the growth process itself.

All possible configurations which are renormalized to a bulk bond are shown in Fig. 1(b). $W_{\alpha}$ is the weight of configuration $\alpha$ to appear in the SPA cluster. Similar to the relations between $C_{\alpha}$, we obtain the following relations for $W_{\alpha}$ 's:

$$
W_{0}=0.5 C, \quad W_{1}=0.5 C \text {. }
$$

Based on the length and time scale invariance of the cluster, we expect that the weights $C_{\alpha}$ 's and $W_{\alpha}$ 's are invari-

TABLE II. Values of $C_{\alpha}$ and $p_{\alpha, i}(3 \times 3$ cell $)$.

\begin{tabular}{ccccccc}
\multicolumn{6}{c}{ TABLE II. Values of $C_{\alpha}$ and $p_{\alpha, i}(3 \times 3$ cell $)}$. \\
\hline \hline$\alpha$ & $C_{\alpha}$ & $p_{\alpha, 1}$ & $p_{\alpha, 2}$ & $p_{\alpha, 3}$ & $p_{\alpha, 4}$ & $p_{\alpha, 5}$ \\
\hline 0 & 0.116696 & 1 & 0 & 0 & 0 & 0 \\
1 & 0.116696 & 1 & 0 & 0 & 0 & 0 \\
2 & 0.116696 & $\frac{2}{3}$ & $\frac{1}{3}$ & 0 & 0 & 0 \\
3 & 0.116696 & $\frac{2}{3}$ & $\frac{1}{6}$ & $\frac{1}{6}$ & 0 & 0 \\
4 & 0.077797 & $\frac{1}{3}$ & $\frac{2}{3}$ & 0 & 0 & 0 \\
5 & 0.038899 & $\frac{1}{3}$ & $\frac{1}{3}$ & $\frac{1}{3}$ & 0 & 0 \\
6 & 0.038899 & $\frac{1}{3}$ & $\frac{1}{3}$ & $\frac{1}{3}$ & 0 & 0 \\
7 & 0.077797 & $\frac{1}{3}$ & $\frac{1}{2}$ & $\frac{1}{6}$ & 0 & 0 \\
8 & 0.103730 & $\frac{1}{3}$ & $\frac{1}{3}$ & $\frac{1}{3}$ & 0 & 0 \\
9 & 0.025932 & $\frac{1}{3}$ & $\frac{5}{18}$ & $\frac{5}{18}$ & $\frac{1}{9}$ & 0 \\
10 & 0.038899 & $\frac{1}{3}$ & $\frac{1}{3}$ & $\frac{1}{6}$ & $\frac{1}{6}$ & 0 \\
11 & 0.012966 & $\frac{1}{3}$ & $\frac{1}{3}$ & $\frac{1}{3}$ & 0 & 0 \\
12 & 0.034577 & $\frac{1}{3}$ & $\frac{1}{3}$ & $\frac{1}{3}$ & 0 & 0 \\
13 & 0.011526 & $\frac{1}{3}$ & $\frac{1}{3}$ & $\frac{1}{6}$ & $\frac{1}{6}$ & 0 \\
14 & 0.013687 & $\frac{1}{3}$ & $\frac{1}{3}$ & $\frac{1}{3}$ & 0 & 0 \\
15 & 0.004322 & $\frac{1}{3}$ & $\frac{1}{6}$ & $\frac{1}{6}$ & $\frac{1}{6}$ & $\frac{1}{6}$ \\
16 & 0.013687 & $\frac{1}{3}$ & $\frac{1}{3}$ & $\frac{1}{3}$ & 0 & 0 \\
17 & 0.012966 & $\frac{1}{3}$ & $\frac{1}{3}$ & $\frac{1}{6}$ & $\frac{1}{6}$ & 0 \\
18 & 0.003362 & $\frac{1}{3}$ & $\frac{1}{3}$ & $\frac{1}{6}$ & $\frac{1}{6}$ & 0 \\
19 & 0.003842 & $\frac{1}{3}$ & $\frac{1}{3}$ & $\frac{1}{9}$ & $\frac{1}{9}$ & $\frac{1}{9}$ \\
20 & 0.006723 & $\frac{1}{3}$ & $\frac{1}{3}$ & $\frac{1}{3}$ & 0 & 0 \\
21 & 0.003362 & $\frac{1}{3}$ & $\frac{1}{3}$ & $\frac{1}{6}$ & $\frac{1}{6}$ & 0 \\
22 & 0.006723 & $\frac{1}{3}$ & $\frac{1}{3}$ & $\frac{1}{3}$ & 0 & 0 \\
23 & 0.000987 & $\frac{1}{3}$ & $\frac{1}{3}$ & $\frac{1}{3}$ & 0 & 0 \\
22 & 0.000560 & $\frac{1}{3}$ & $\frac{1}{3}$ & $\frac{1}{3}$ & 0 & 0 \\
25 & 0.001974 & $\frac{1}{3}$ & $\frac{1}{3}$ & $\frac{1}{3}$ & 0 & 0 \\
\hline \hline & & & & & & \\
\hline
\end{tabular}




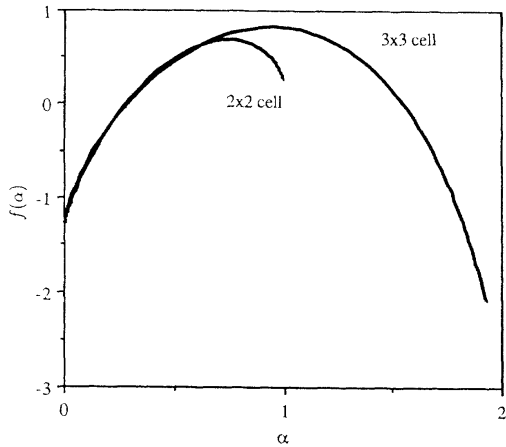

FIG. 3. The plot of $f$ vs $\alpha$ for the SPA on the square lattice for $2 \times 2$ and $3 \times 3$ cell renormalizations.
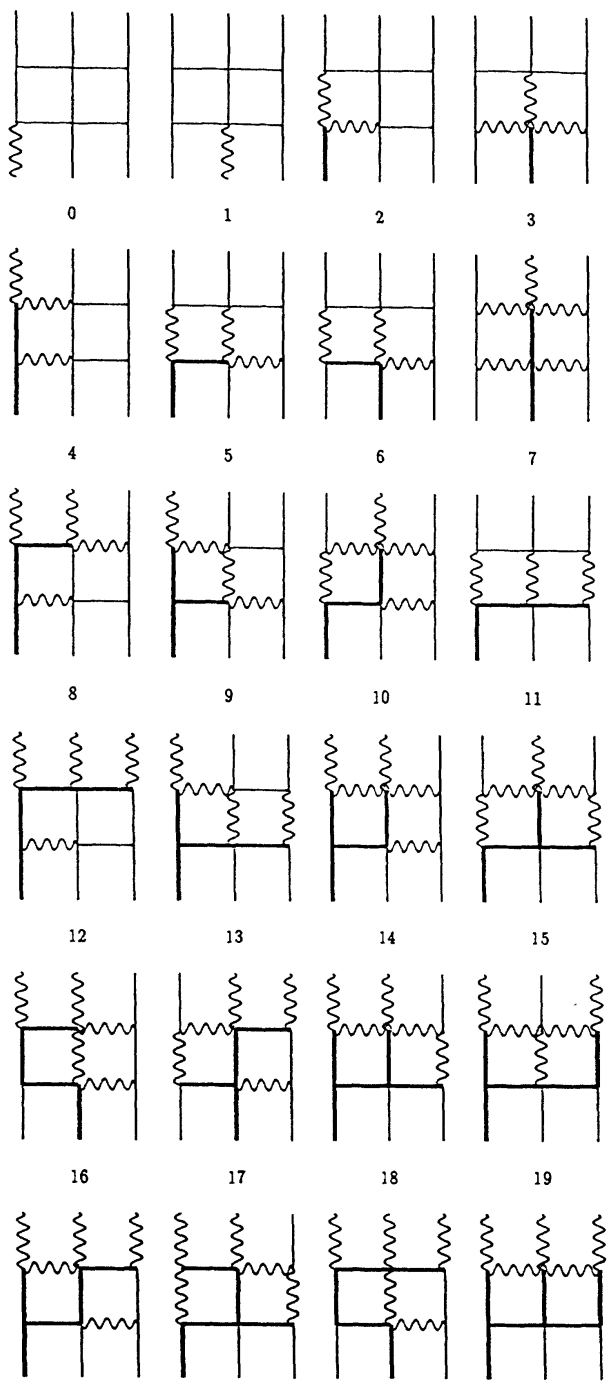

20

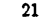

22

23

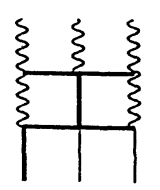

24

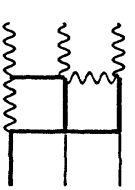

25

FIG. 4. All possible configurations which are renormalized to the vertical perimeter bonds for the square lattice in a $3 \times 3$ cell.

ant under our RG transformation. Therefore we derive the recursion relation for the masses $M_{n}$ and $m_{n}$ : from Fig. 1,

$$
\begin{aligned}
& m_{n}=\left(C_{0}+C_{1}+2 C_{2}\right) m_{n-1}+\left(C_{1}+C_{2}\right) M_{n-1}, \\
& M_{n}=\left(2 W_{0}+2 W_{1}\right) M_{n-1}+W_{1} m_{n-1} .
\end{aligned}
$$

From Eqs. (3a) and (4b), we obtain the recursion relations,

$$
\begin{aligned}
& m_{n}=1.2 m_{n-1}+0.6 M_{n-1}, \\
& M_{n}=2 M_{n-1}+0.5 m_{n-1} .
\end{aligned}
$$

From the largest eigenvalue $\lambda_{+}$, we find the fractal dimension of the cluster:

$$
D_{f}=\ln \left(\lambda_{+}\right) / \ln (2)=[\ln (2.28) / \ln (2)]=1.19 \text {. }
$$

From the probabilities $C_{\alpha}$ of configurations $\alpha$ presented in Fig. 1(a) and the growth probabilities $p_{\alpha, i}$ at perimeter bonds, the hierarchical dimensions of Halsey et al. $[4,10]$ are in our case

$$
\begin{aligned}
& D(q)=-\frac{\ln \left(0.4+1.2 \times 2^{-q}\right) / \ln (2)}{q-1} \quad(q \neq 1), \\
& D(1)=0.6 .
\end{aligned}
$$

The resulting $D(q)$ is shown in Fig. 2. It is a monotonic
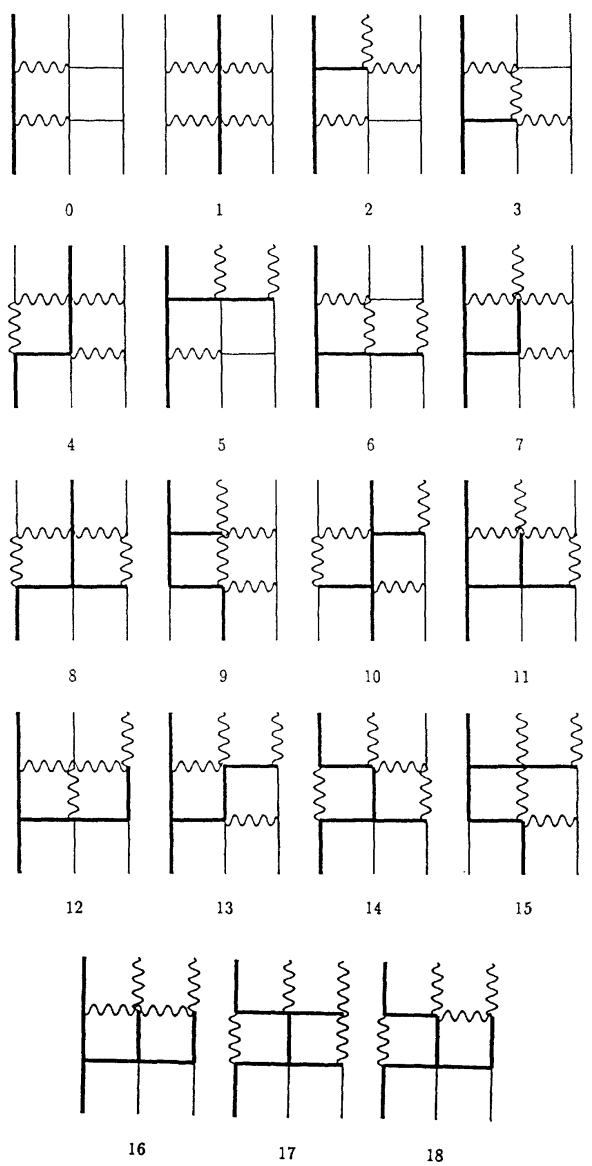

FIG. 5. All possible configurations which are renormalized to the vertical bulk bonds for the square lattice in a $3 \times 3$ cell. 
curve consistent with the inequality [11] $D(q)<D\left(q^{\prime}\right)$ when $q>q^{\prime}$. The $f-\alpha$ spectrum [10] is given by the Legendre transformation of $D(q)$,

$$
\begin{aligned}
& \partial f / \partial q=q(\partial \alpha / \partial q), \\
& \partial[(q-1) D(q)] / \partial q=\alpha(q) .
\end{aligned}
$$

The result is shown in Fig. 3. It is a convex curve, as expected.

Similarly, $3 \times 3$ cell renormalization of the SPA on the square lattice can be carried out directly. Then all the configurations which are renormalized to the vertical perimeter bond and the vertical bulk bond are shown in Figs. 4 and 5. The values of $C_{\alpha}$, growth probabilities $p_{\alpha, i}$ on the external perimeter bonds (Table II), and $W_{\alpha}$ can be found from

$$
\begin{aligned}
& C_{1}=C_{0}, C_{2}=C_{0}, \quad C_{3}=C_{0}, C_{4}=0.6667 C_{0}, \\
& C_{5}=0.6667 C_{0}, \quad C_{6}=0.6667 C_{0}, C_{7}=0.8889 C_{0}, \\
& C_{8}=0.2222 C_{0}, \quad C_{9}=0.3333 C_{0}, C_{10}=0.1111 C_{0}, \\
& C_{11}=0.2963 C_{0}, \quad C_{12}=0.0988 C_{0}, \quad C_{13}=0.1173 C_{0}, \\
& C_{14}=0.0370 C_{0}, C_{15}=0.1173 C_{0}, \quad C_{16}=0.1111 C_{0}, \\
& C_{17}=0.0288 C_{0}, \quad C_{18}=0.0329 C_{0}, \quad C_{19}=0.0576 C_{0},
\end{aligned}
$$

$C_{20}=0.0288 C_{0}, \quad C_{21}=0.0576 C_{0}, C_{22}=0.0085 C_{0}$,

$C_{23}=0.0048 C_{0}, \quad C_{24}=0.0169 C_{0}, \quad \sum_{\alpha} C_{\alpha}=1$.

$W_{0}=0.2222 C, \quad W_{1}=0.2222 C, \quad W_{2}=0.5926 C$,

$W_{3}=0.0741 C, W_{4}=0.1111 C, W_{5}=0.2963 C$,

$W_{6}=0.0329 C, \quad W_{7}=0.0782 C, \quad W_{8}=0.0123 C$,

$W_{9}=0.0782 C, W_{10}=0.0741 C, W_{11}=0.0192 C$,

$W_{12}=0.0219 C, W_{13}=0.0576 C, W_{14}=0.0192 C$,

$W_{15}=0.0576 C, \quad W_{16}=0.0085 C, W_{17}=0.0048 C$,

$W_{18}=0.0168 C, \sum_{\alpha} W_{\alpha}=1$.

Therefore, from the mass conservation, the recursion relations are

$$
\begin{aligned}
& m_{n}=1.4954 m_{n-1}+1.2536 M_{n-1}, \\
& M_{n}=1.1792 M_{n-1}+3.1054 m_{n-1},
\end{aligned}
$$

with eigenvalues $\lambda_{+}=3.7586$ and $\lambda_{-}=0.8422$. Therefore the fractal dimension is $D_{f}$ $=\ln (3.7586) / \ln (3.0)=1.205$, which is in surprisingly good agreement with simulation value 1.21 [4].

The hierarchical dimensions are given by

$$
\begin{aligned}
& \begin{aligned}
D(q)= & -\ln \left[0.233+0.311 \times\left(\frac{2}{3}\right)^{q}+0.078 \times 2^{-q}+1.271 \times 3^{-q}+0.052 \times\left(\frac{5}{18}\right)^{q}\right. \\
& \left.+0.469 \times 6^{-q}+0.038 \times 9^{-q}\right] /[(q-1) \ln (3)] \quad(q \neq 1),
\end{aligned} \\
& D(1)=0.677,
\end{aligned}
$$

which are shown in Fig. 2, and the $f$ - $\alpha$ spectrum is shown in Fig. 3. The surprising overlap of the curves in both Figs. 2 and 3 in the large- $q$ (small- $\alpha$ ) region is a strong sign in favor of the approach. There is no reason to expect the good convergence of the $D(q)-q$ and $f-\alpha$ curves of finite-size RG calculations for small and negative $q$ where the extremely small growth probability region will dominate the value of $D(q)$. In fact, the clear deviation of $D(q)-q$ and $f-\alpha$ curves of $3 \times 3$ cell RG calculation from those of $2 \times 2$ cell $R G$ calculation is consistent with the suggestion that the multifractal concept may not be applicable for negative $q$ in fractal aggregation [12-14].

To summarize, we have applied the KRG method of Wang, Shapir, and Rubinstein to the SPA deposition pro- cess in a two-dimensional square lattice. Our value $D_{f}=1.205$ is to be compared with the square-lattice simulation value $D_{f}=1.21 \pm 0.01$ [4]. We have also calculated the multiscaling structure of the shortest-path aggregation. We find that the values of multifractal dimensions, such as $f(\alpha)$, from $2 \times 2$ and $3 \times 3$ cell RG calculations agree with each other very well for small $\alpha$ (large $q$ ), while they deviate with each other at large $\alpha$ (small and negative $q$ ). The further simulation of the SPA model on the multifractal dimensionality $D(q)$ will be useful in order to test the theory. The similar calculations on three and higher dimensions are straightforward, and it will be interesting to carry out such calculations and compare with corresponding simulation results.
[1] T. Visek, Fractal Growth Phenomena (World Scientific, Singapore, 1989).

[2] T. A. Witten and L. M. Sander, Phys. Rev. Lett. 47, 1400 (1981); Phys. Rev. B 27, 2686 (1983).

[3] S. Liang and L. P. Kadanoff, Phys. Rev. A 31, 2628 (1985); Phys. Lett. 102A, 38 (1984).

[4] X. R. Wang, Phys. Rev. A 40, B6767 (1989); X. R. Wang and X. F. Wang, ibid. 45, 1274 (1992).

[5] M. Kardar, G. Parisi, and Y. C. Zhang, Phys. Rev. Lett. 56, 889 (1986); Y. Shapir, J. Phys. A 18, L897 (1985); Y. Shapir and Y. C. Zhang, J. Phys. Lett. 46, L529 (1985).

[6] X. R. Wang et al., Phys. Rev. A 39, 5974 (1989); J. Phys. A 22, L507 (1989); Phys. Lett. A 138 (1989).

[7] H. Gould et al., Phys. Rev. Lett. 50, 686 (1983); N. Nakan- ishi and F. Family, Phys. Rev. A 32, 3606 (1985).

[8] T. Nagatani, J. Phys. A 20, L381; 20, L623 (1987).

[9] X. Wang and Y. Huang, Phys. Rev. A 46, 5038 (1992).

[10] T. C. Halsey et al., Phys. Rev. A 33, 1141 (1986); C. Amitrano et al., Phys. Rev. Lett. 57, 1016 (1986).

[11] H. G. E. Hentschel and I. Procaccia, Physica D 8, 435 (1983).

[12] A. Aharony, Tel Aviv University Report No. TAUP1778-90, 1990 (unpublished).

[13] J. Lee and H. E. Stanley, Phys. Rev. Lett. 61, 2945 (1988).

[14] M. H. Jensen, in Universalities in Condensed Matter, edited by R. Jullien, L. Peliti, R. Rammal, and N. Boccara (Springer, Heidelberg, 1988), p. 233. 